\title{
Analyse spatio-temporelle de trajets propagés en ASM sur de moyennes distances
}

\author{
B. FAURE, D. MAUUARY et T. TERRE*
}

CEPHAG, INPG/ENSIEG, URA 346 du CNRS, BP. 46, 38402 Saint Martin d'Hères cedex, France

* LPO, IFREMER, BP. 70, 29280 Plouzane cedex, France

\begin{abstract}
We propose, from experimental data, to identify the ocean acoustic multipaths, between acoustic sources and receivers separated about $100 \mathrm{~km}$. After the description of experiment, we show that the active beamforming, using the knowledge of emitted signal, leads to satisfying spatial identification when the multipaths are temporarily separed, in spite of an antenna with only two phase centers. A fast spatio-temporal localization algorithm is used and useful results for acoustic tomography are obteined.
\end{abstract}

\section{INTRODUCTION}

Cette présentation entre dans le cadre des études de tomographie acoustique océanique pour lesquelles il est important d'identifier, aussi bien en temps que spatialement, les rayons se propageant dans le milieu marin entre des émetteurs et des récepteurs distants de quelques $100 \mathrm{~km}$. Habituellement la séparation angulaire des rayons reçus est obtenue en utilisant une antenne de réception et en effectuant des traitements appropriés. Dans le cadre précis de l'expérimentation support de cette étude, nous ne disposons que de deux centres de phase à la réception, ce qui limite la résolution angulaire donnée par la formation de voies. Toutefois, en utilisant la connaissance du signal émis et en réalisant une formation de voies active, ce qui revient à exploiter la phase de la fonction d'intercorrélation des signaux des deux centres de phase aux instants ou le signal est présent, nous montrons qu'il est possible de déterminer l'angle d'incidence des rayons reçus avec une résolution angulaire précise de quelques degrés naturellement fonction du rapport signal à bruit.

\section{CONTEXTE EXPERIMENTAL}

Dans le cadre du programme Européen de tomographie acoustique océanique THETIS supporté par un projet MAST, une expérimentation en mer a été programmée et organisée par les différents partenaires. Lors de cette campagne : CONVHIV, qui s'est déroulée en Méditerranée durant l'année 1992, le signal émis par chaque instrument était constitué de 14 séquences de signaux BPSK de durée de 5.11 secondes chacune, séparés par des silences de $2 \mathrm{~h} 24$. Les instruments étaient immergés à $-150 \mathrm{~m}$ et distants de plusieurs dizaines de $\mathrm{km}$. A la réception, le signal est reçu sur une antenne [1] constituée de deux sousantennes dont les centres de phase sont distants d'une demi longueur d'onde (fréquence centrale d'émission à $400 \mathrm{~Hz}$ ). Il est à noter que les signaux disponibles sont les signaux reçus après démodulation complexe à la fréquence de la porteuse. Parmi les couples d'émetteurs récepteurs, nous avons analysé tout d'abord la paire dont la distance de propagation est de $138 \mathrm{~km}$, (pour cette configuration géométrique, les signaux étaient directement reçus sur l'antenne sans moyennage temporel à l'intérireur des instruments) ; puis nous avons traité les signaux directement enregistrés dans les mouillages pour un couple séparé de $52 \mathrm{~km}$, (pour cette analyse, les signaux étaient moyennés sur la durée de la séquence d'émission). 


\section{LOCALISATION PAR FORMATION DE VOIES}

La première exploitation de l'antenne consiste à effectuer une formation de voies classique, en passif, en utilisant les signaux en temps des deux centres de phase de l'antenne réduite. Le schéma de traitement est donné ci dessous.

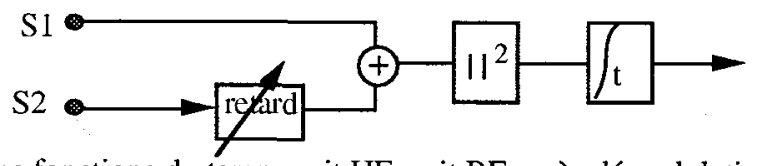

S1 et S2 sont les signaux reçus fonctions du temps soit HF, soit BF après démodulation complexe.

Il va de soit qu'avec l'antenne utilisée, la résolution angulaire $\left(=60^{\circ}\right)$ ne permet pas la localisation des trajets.

\section{LOCALISATION SPATIO-TEMPORELLE}

\subsection{Formation de voies active}

La connaissance du signal d'émission permet d'avoir une stratégie différente en ce qui concerne la localisation angulaire en faisant conjointement une localisation temporelle. En effet, il est possible de réaliser une formation de voies entre les deux centres de phase et de calculer ensuite lintercorrélation du signal de sortie avec la référence d'émission. Le schéma de traitement est alors le suivant:

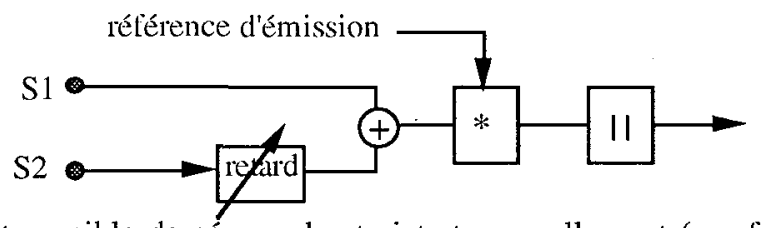

Grâce à ce traitement, il est possible de séparer les trajets temporellement (par filtrage adapté) mais également spatialement (par formation de voies). La figure 1 est la représentation bi-dimentionnelle retard de propagation-angles d'arrivée (en courbe de niveau après moyenne sur les 14 séquences reçues). La séparation temporelle des trajets est nettement visible quant à la résolution angulaire elle n'est pas très précise comme prévu mais on constate des directions d'arrivée privilégiées pour les diflérents trajets.

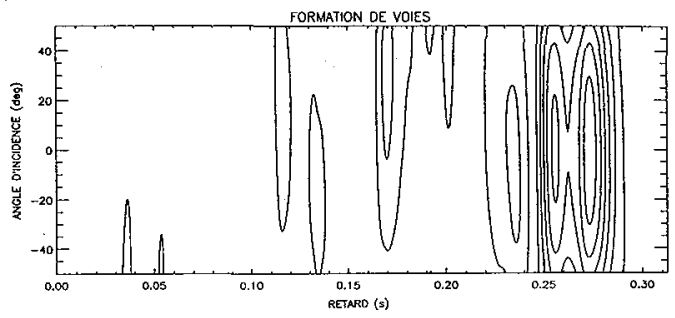

Figure 1: représentation spatio-temporelle des différents trajets de propagation

\subsection{Exploitation du module}

Le diagramme de directivite de l'antenne ayant un lobe principal large, c'est-a-dire une résolution angulaire faible, nous nous sommes attachés dans un premier temps à déterminer non pas l'angle d'arrivée des rayons acoustiques mais uniquement le signe de l'angle. Pour ce faire, deux voies sont formées correspondant à des angles de pointage de l'antenne de +15 et -15 degrés. Ce choix est fortement conditionné par l'aspect tomographie du problème pour lequel seuls les rayons réfractés et réflechis surface sont utilisés. L'utilisation des rayons réfléchis au fond reste conditionné par la connaissance précise de la bathymétrie et de la nature du terrain.

La figure 2 présente les résultats obtenus avec les données acquises durant 7 jours dans les instruments après une une formation de voies exhaustive. Le tracé de gauche est le suivi au cours du temps de la réponse impulsionnelle du milieu. Chaque trajet est identifié par le module de la fonction d'intercorrélation, de plus le signe de l'angle d'arrivée est matérialisé par des croix lorsqu'il est positif et des ronds pleins 
lorsqu'il est négatif. Sur le tracé de droite, nous avons effectué l'analyse spatiale au cours du temps du premier et du dernier rayon. Le premier était déclaré positif, on le trouve aux alentours de $8^{\circ}$, le dernier rayon présumé négatif se situe effectivement en moyenne vers $-15^{\circ}$. Les valeurs trouvées sont très proches des valeurs prédites par le modèle géométrique (trait plein à -19 et $8^{\circ}$ ).
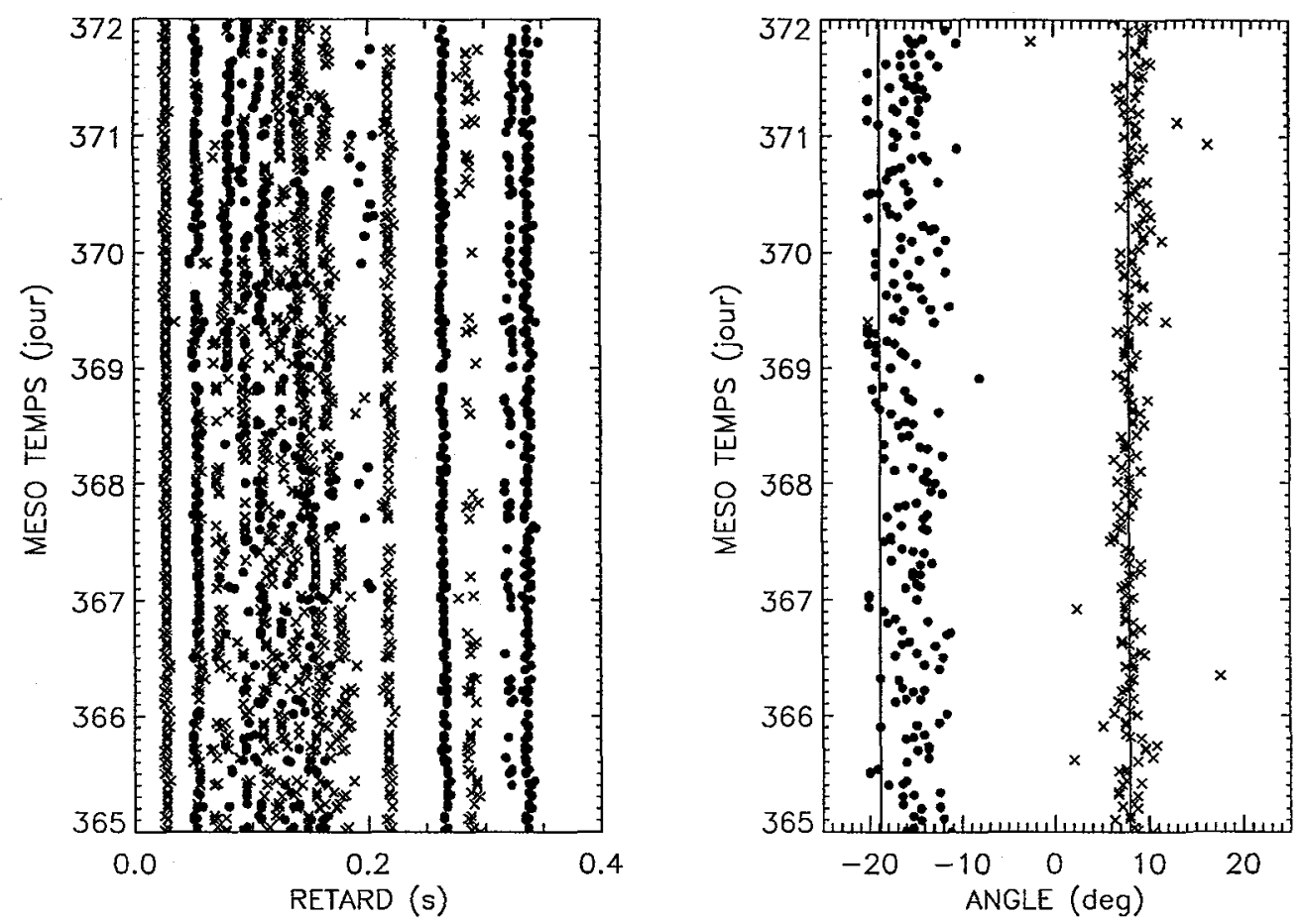

Figure 2 : à gauche : suivi des trajets positifs $(x)$ ou négatifs $(\bullet)$ de la réponse impultionnelle du milieu à droite : suivi angulaire du premier et du dernier rayon.

\subsection{Exploitation de la phase par algorithme rapide}

L'inconvéniant majeur du traitement par formation de voies active est le coût important de calcul. Pour éviter cela, nous proposons un traitement en deux temps. Tout d'abord nous effectuons la localisation temporelle des différents rayons en analysant de façon indépendante les deux voies reçues. Cette localisation est faite selon la méthode classique de l'intercorrélation entrée sortie lorsque le signal émis est du type large bande comme c'est le cas ici. Il est alors aisé de localiser la présence des différents rayons sur les deux voies de l'antenne. Puis en mesurant la phase de la fonction d'intercortélation aux instants ou le signal est présent sur les deux voies, on peut mesurer l'écart de phase relatif et il est ensuite facile de transposer cette information de phase en intormation angulaire. Compte tenu de la géométrie de l'antenne, l'angle d'incidence d'un rayon est donné par la relation : $\theta=\arcsin \Delta \varphi / \pi$. le schéma de traitement proposé est le suivant :

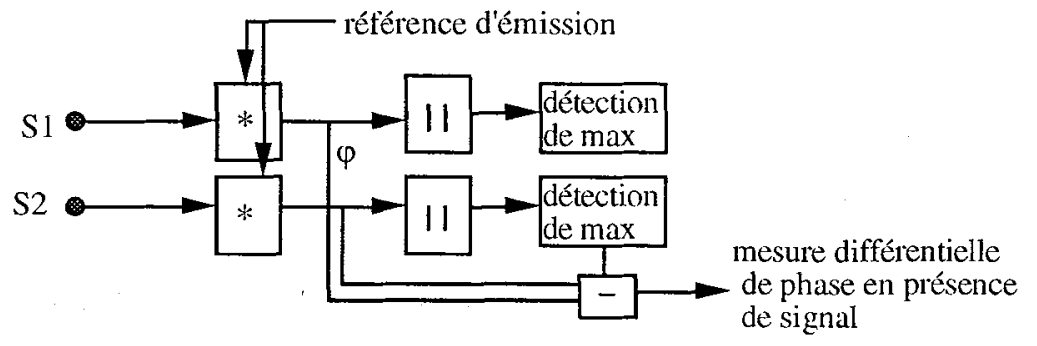


On peut utiliser la présence, à l'émission, de la succession des motifs BPSK pour améliorer la résolution temporelle, par moyennage sur les récurrences, mais également la résolution angulaire en moyennant les informations de phase sous le pic d'intercorrélation.

Toutefois, le rapport signal à bruit mesuré sur le module de la fonction d'intercorrélation a une grande incidence sur l'erreur de l'estimation de l'angle. En effet, nous avons tracé voir figure 3 la courbe donnant les barres d'erreur sur l'estimation des mesures de phase en fonction du rapport signal à bruit en entrée. Les résultats obtenus sont présentés sur la figure 4 ou nous avons tracé les réponses impultionnelles des 2 voies exprimées en $\mathrm{dB}$ et en regard nous montrons la localisation spatiale obtenue par la mesure de phase. Nous avons superposé pour chaque mesure les barres d'erreur liée au rapport signal à bruit en entrée.

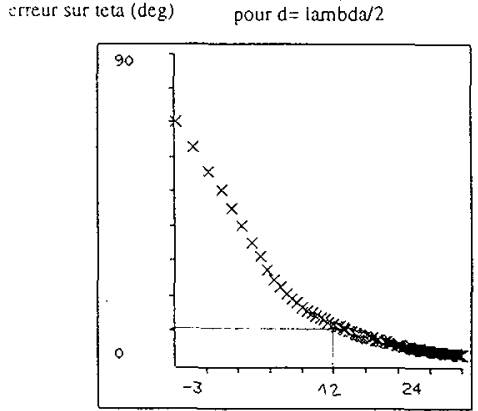

rapport $S / B(d B)$

Figure 3 : tracé de l'erreur d'estimation de l'angle d'arrivée en fonction du rapport S/B du signal reçu

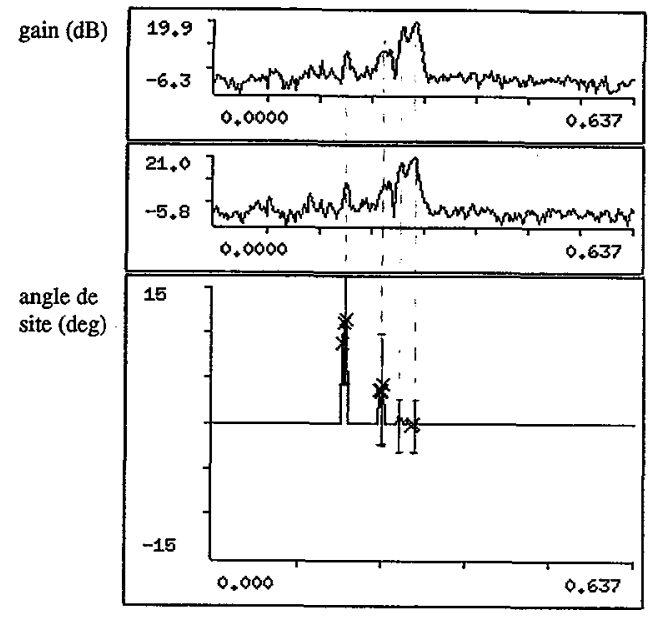

retard (s)

Figure 4 : réponse spatio-temporelle des principaux rayons de propagation

a) et b) réponse temporelle mesurée sur chaque voie de réception

c) localisation angulaire par mesure comparative de phase des principaux rayons.

\section{CONCLUSIONS}

Malgré l'utilisation d'une antenne de réception constituée uniquement de deux centres de phase, nous avons montré sur des données expérimentales qu'il était possible d'identifier de façon spatiale et temporelle les trajets de propagation. La méthode d'analyse proposée qui est basée sur la formation de voies active peut etre exploitée soit en considérant l'aspect énergétique ou bien en analysant l'information de phase relative à chaque voie. Nous avons proposé un algorithme rapide qui permet l'analyse spatiale lorsqu'il y a présence de signal donc trajet de propagation. Les résultats obtenus sont en parfait accord, lorsque le rapport signal à bruit est suffisant et lorsque les trajets sont isolés, avec les prédictions du modèle géométrique. La discrimination et le suivi au cours du temps des rayons de propagation aaussi bien dans le plan temporel que spatial peut être un avantage certain en tomographie acoustique océanique ou en communication.

\section{Remerciements}

Le projet THETIS-I est financé par le programme Marine Science and Technology (MAST) de la Communaute Europeenne, DG XII sous le contrat MAST-0008C.

\section{Bibliographie}

Faure B. Etude d'une antenne verticale pour la discrimination des angles d'arrivée des signaux en tomographie acoustique océanique. Rapport Cephag n ${ }^{\circ} 60 / 90$

Peter F. Worcester An example of ocean acoustic multipath identification at long range using both travel time and vertical arrival angle. JASA 70 (6), Dec 1981 